\title{
ANALITICIDADE E INFERÊNCIA MATERIAL: OBSERVAÇÕES À TEORIA DA INFORMAÇÃO SEMÂNTICA DE CARNAP E BAR-HILLEL
}

\author{
Ralph Leal Heck ${ }^{1}$ \\ Universidade Estadual do Ceará e Faculdade Católica de Fortalez \\ imagmundi@hotmail.com
}

\begin{abstract}
Resumo
O presente artigo tem por objetivo apresentar duas observações à teoria da informação semântica de Carnap e BarHillel, primeiro, que as sentenças tomadas por verdadeiras em L deveriam ser tomadas como informativas e não deveriam estar na mesma classe que as tautologias e a segunda crítica é que estas sentenças tem a capacidade de expressar nossos quadros conceituais na forma de restrições. Isto significa que o valor informativo desta classe de sentenças deve ser interpretado como versando sobre nossas inferências materiais. Para realizar esta tarefa, iniciarei apresentando o conceito de informação, seus principais paradigmas e o tipo de teoria na qual se enquadra a teoria de Carnap e Bar-Hillel. Em seguida, farei a exposição das principais funções medidoras de informação, seguido de exemplos ilustrativos. E, por fim, irei executar um conjunto de críticas que culminam na reavaliação do papel que as sentenças verdadeiras em L desempenham.
\end{abstract}

Palavras-chave: Informação Semântica. Analiticidade. Inferência Material.

\section{ANALYTICITY AND MATERIAL INFERENCE: REMARKS TO THE CARNAP AND BAR-HILLEL SEMANTIC INFORMATION THEORY}

\begin{abstract}
This article aims to present two remarks to the Carnap and Bar-Hillel semantic information theory, first that sentences taken as true in L should be taken as informative and should not be in the same class as tautologies and the second remark is that these sentences have the ability to express our conceptual frameworks in the form of constraints. This means that the informative value of this class of sentences should be interpreted as referring to our material inferences. To accomplish this task, I will start by presenting the concept of information, its main paradigms and the kind of theory in which the theory of Carnap and Bar-Hillel fits. I will then present the main measuring functions of information, followed by illustrative examples. And finally, I will execute a set of criticisms that culminate in the re-evaluation of the role that the true sentences in L play.
\end{abstract}

Keywords: Semantic Information. Analyticity. Material Inference.

${ }^{1}$ Doutorando em Filosofia pela Universidade Federal do Ceará; Professor da Universidade Estadual do Ceará e Faculdade Católica de Fortaleza 


\section{INTRODUÇÃO}

Esta sessão destina-se a uma introdução ao conceito de informação, seus paradigmas contemporâneos e à delimitação do tipo de teoria da informação que será trabalhada ao longo do texto.

A palavra 'Informação' é uma expressão polissêmica, e opera como explicandum associado a várias descrições, em diferentes teorias, de acordo com o nível de abstração e de requisitos do contexto em que a palavra se vincula. Segundo Shannon:

À palavra "informação" foram dados diferentes significados, por vários escritores no campo amplo da teoria da informação. É provável que, ao menos, certo número destas abordagens se provará suficientemente útil em certas aplicações, para merecerem estudos posteriores e reconhecimento permanente. Dificilmente espera-se que um único conceito de informação dê conta satisfatoriamente das inúmeras aplicações possíveis neste campo amplo (SHANNON, 1993, p. 180).

Em razão deste contexto plural, ocorreu a tentativa de agrupar as teorias da informação, de modo a sistematizar o conceito. Segundo Adriaans e van Benthem, há três tipos de cenários paradigmáticospara a teoria da informação:

(A) O típico ambiente baseado em lógica permite que um agenteadquirira novas informações sobre como éo mundo real, através de atos de observação, comunicação linguística, ou dedução. [...] o paradigma formal para a teoria é a lógica matemática ou computacional.

(B) Em contraste, o cenário típico de Shannon é sobre uma fonte de emissão de sinais em determinadas frequências, com, digamos, uma "linguagem" vista como um produtor de texto global, e a informação que o receptor capta a partir do emissor é medida em termos de redução de incerteza esperada. [...] Por fim, os paradigmas matemáticos da teoria são a teoria da probabilidade e a física. [...]

(C) Em seguida, tomamos o cenário básico de Kolmogorov. Recebemos uma string de código, e nos perguntamos por seu valor informativo. A resposta é a complexidade algorítmica da string, definida como o tamanho domenor programa que calcula-o em uma máquina de Turing universal fixa. [...] Assim, surge o seguinte "intercâmbio": Informação-B inicia com a noção de probabilidade como fundamental e deriva um código ideal. Informação-C começa com a noção de código mais curto como fundamental e deriva uma probabilidade a priori a partir dele (ADRIAANS; VAN BENTHEM, 2008, p.15-16).

O paradigma (C) é exemplificado por uma teoria da informação algorítmica. A principal característica da teoria é a ideia de que informação tem a ver com o trabalho útil que um algoritmo faz para produzir um resultado e que este trabalho pode ser mensurado com base no comprimento da descrição do algoritmo. Assim, o conteúdo informativo de uma string gerada é o comprimento em bits do programa mais curto (também chamado de programa elegante) usado para produzir a string, igualmente referido por sua complexidade. Uma string finita s pode ser produzida com o comprimento $|s|+|c|$, onde $c$ é o comprimento em bits do código mínimo para produzirs. Este paradigma computacional originou-se de (B),o cenário da teoria da informação de Shannon. Sua teoria tem origem no setor de comunicações elétricas e nasceu 
da investigação por um modelo estatístico do funcionamento dos equipamentos de transmissão e recepção de sinais. Entretanto, o modelo criado não se restringe ao fluxo de sinais elétricos. Ele pode ser aplicado em contextos abstratos, contendo como meio de transmissão de informação coisas como: palavras, figuras, música, etc. Neste sentido, a ocupação principal do modelo de Shannon é identificar condições eficientes para a transmissão de informação e entender informação como um sinal capaz de reduzir a probabilidade dos próximos sinais a serem emitidos, dada determinada distribuição estatística não-uniforme de ocorrência destes sinais sobre um sistema (léxico) de sinais possíveis.

Ambos os paradigmas comportam uma visão sintática e probabilística do conceito de informação, interpretando-a como um fenômeno eminentemente físico-probabilístico.É apenas no paradigma (A) que as noções de significado e verdadese encontram essencialmente ligadas ao conceito de informação, tendo como seus critérios de importância o significado, a sobredade (aboutness), a relevância, a confiabilidade ou a utilidade da informação.Este é o paradigma para a teoria da informação semântica.A informação semântica pode ser dividida em duas classes: 1) informação instrucional, como gatilho para a execução de uma ação e 2) informação factual, fragmento da comunicação que informa um fato.

A primeira classe diz respeito à pragmática da linguagem, às ações que decorrem do fluxo de informação entre emissores e receptores. A esta classe de informação semântica, não é sempre possível atribuir valores de verdade. Informações que expressem ordens, perguntas, instruções nãopodem ser associadas inequivocamente a valores de verdade, mas, de modo algum se provam inúteis ou dissociadas de valores semânticos. A argumentação final deste artigopretende mostrar que existe uma dimensão pragmática de fundo que orienta a informação representacional e vero-funcional, da linguagem.

A segunda classe, historicamente a primeira a ser desenvolvida no paradigma (A), associa-se aos valores de verdade. Toda informação factual possui um conteúdo semântico de verdade (verdadeiro, falso, não-verdadeiro, etc), este conteúdo semântico, em especial, para alguns autores, o valor semântico 'verdadeiro', é condição necessária para a construção do conhecimento.Aqui, podemos seguir Luciano Floridi (FLORIDI, 2011, p. 108-117) e distinguir entre dois modos de definir a relação da informação com os valores de verdade: A abordagem semântica fraca (TWSI) e a abordagem semântica forte da informação (TSSI).A TWSI postula a independência das unidades de informação (ínfons) em relação aos valores de verdade. Nessa concepção, uma proposição $p$ carrega informação, na medida em que modela $w$ (uma situação informada), segundo o princípio de relacionamento inverso (IRP), que diz: quanto mais casos possíveis forem excluídos com a informação $p$, mais informativa $p$ é.Isto significa que sentenças 
falsas podem ser informativas, mas não tão informativas quanto sentenças verdadeiras.Nesta perspectiva, as sentenças falsas excluem uma classe de estados de coisas possíveis. Por exemplo: suponha que eu esteja vendo a face monocromática de um cartão na mão de outra pessoa, que não sabe a cor da face voltada para mim. Se perguntado por ela pela cor do cartão, quando digo que é falso que o cartão seja vermelho, apenas a cor vermelha é excluída de uma longa lista de cores possíveis que o cartão pode assumir. Se digo que é verdadeiro que o cartão é azul, todas as outras opções de cores são excluídas automaticamente.

Entretanto, como aponta Floridi (2011, p.108-117), se aceitarmos que toda sentença falsa seja informativa, topamos com o paradoxo Bar-Hillel-Carnap (BCP), que diz:sentenças logicamente contraditórias excluem todos os casos possíveis, logo, são interpretadas pelo IRP como completamente informativas(mais informativas que sentenças verdadeiras) esendo completamente informativas, deveriam ser aceitáveis.

$\mathrm{Na}$ TSSI, os valores de verdade desempenham um papel necessário (definíveis a priori). Tal que, a quantidade de informação contida em uma sentença é dada, não pela quantidade de situações possíveis excluídas, mas pela relevância que uma dada informação possui, em dado contexto de aplicação. Ou seja, a teoria abre mão do IRP, e associa o valor informativo de suas sentenças a um intervalo de valores que variam desde completamente falsa em todas as situações e completamente verdadeira em todas as situações. Às duas condições limite acima, a teoria classifica como contradição e tautologia, respectivamente. As sentenças que se encontram entre estes extremos são classificadas como contingentes (FLORIDI, 2011, p.117121).

\section{A TEORIA DA INFORMAÇÃO SEMÂNTICA DE CARNAP E BAR-HILLEL}

Nesta sessão será feita uma apresentação da primeira teoria da informação semântica, suas principais funções de medição, acompanhadas de exemplos e, para por fim, fazer críticas à visão de que sentenças analíticas não são informativas.

A teoria da informação semântica esboçada por Carnap e Bar-Hillelé a teoria que Floridi designa por TWSI, sendo também referida na literatura como teoria clássica da informação semântica.Seu ponto de partida é a ideia de que há um sentido útil e quantitativo, no qual o conteúdo semântico de sentenças verdadeiras ou falsas pode ser entendido como um tipo de informação, relativamente, ao conjunto total de situações por elas modeladas e excluídas (BARHILLEL; CARNAP, 1952. p. 2-3). A teoria deles possui dupla relevância: Eles são os pioneiros 
na formulação de uma teoria da informação semântica (FLORIDI, 2011, p.102-114); E sua teoria é um paralelo com a teoria matemática da comunicação de Shannon(TMC). Vejamos a teoria.

Em primeiro lugar, Carnap e Bar-Hillel (1952), entendem que ao receber uma mensagem, a quantidade de situações excluídas pela asserção contida na mensagem indica o conteúdo informativo da sentença, isto é, a quantidade de informação contida na mensagem.

Para que isto possa ser mensurado, eles definem uma linguagem L, com n constantes e predicados unários, e com os conectivos lógicos habituais. Dadas essas características, o que L pode descrever consistentemente é uma totalidade de estados de descrição (state descriptions) que pode variar exatamente de $2^{\mathrm{n}}$ modos possíveis, cada um desses modos sendo considerado um estado diferente da totalidade. É interessante notar que as constantes individuais são enfatizadas como o ponto de partida da construção das possibilidades que podem ser expressas pela linguagem $\mathrm{L}$.

Primeira medição (pre-sistemática) de informação "In" (com i maiúsculo) indica certas relações lógicas entre sentenças que partilham conteúdo. Relações que podem ser expressas em termos de conjuntos. Um explicatum para In é Cont. Cont é a totalidade dos estados excluídos pelo conteúdo da mensagem. Em outras palavras, se chamarmos o conjunto estados possíveis de $\mathrm{W}$, podemos definir o conteúdo informacional de uma sentença i, em símbolos Cont(i), do seguinte modo:

(1) $\operatorname{Cont}(\mathrm{i})=\{\mathrm{w} \mathrm{W} \mid \mathrm{i}\}$,

Ou seja, o conteúdo de i é o conjunto dos estados de W que falsificam i. Nos casos de uma tautologia $t$ e uma contradição c, temos: (pois nada falsifica uma tautologia) e Cont(c)=W (pois tudo falsifica uma contradição). Ou seja, $t$ é verdadeira para qualquer estado $w$ e $c$ para nenhum estado $w$.

Vejamos um exemplo: Sejam dois indivíduos quaisquer designados respectivamente, pelas constantes individuais $a, b$; e existam dois predicados unários tais que "x é mulher" é designado por e "x é casado" por .

Dizemos que $\mathrm{W}=2^{2.2}=2^{4}=16$. Isto é $\mathrm{W}$ é um conjunto de 16 estados possíveis, que variam na combinação de atribuição dos predicados afirmados ou negados às constantes individuais, conforme a seguinte lista:

(Ex1):

\begin{tabular}{|c|c|c|c|c|l|l|l|l|l|}
\hline $\mathrm{w}$ & \multicolumn{2}{|c|}{$\mathrm{a}$} & \multicolumn{2}{c|}{$\mathrm{b}$} & $\mathrm{w}$ & \multicolumn{2}{|c|}{$\mathrm{a}$} & \multicolumn{2}{|c|}{$\mathrm{B}$} \\
\hline 1 & $M x$ & $C x$ & $M x$ & $C x$ & 9 & $M x$ & $\neg C x$ & $\neg M x$ & $C x$ \\
\hline 2 & $\neg M x$ & $C x$ & $M x$ & $C x$ & 10 & $M x$ & $\neg C x$ & $M x$ & $\neg C x$ \\
\hline 3 & $M x$ & $\neg C x$ & $M x$ & $C x$ & 11 & $M x$ & $C x$ & $\neg M x$ & $\neg C x$ \\
\hline
\end{tabular}




\begin{tabular}{|c|c|c|c|c|c|c|c|c|c|}
\hline 4 & $M x$ & $C x$ & $\neg M x$ & $C x$ & 12 & $\neg M x$ & $\neg C x$ & $\neg M x$ & $C x$ \\
\hline 5 & $M x$ & $C x$ & $M x$ & $\neg C x$ & 13 & $\neg M x$ & $\neg C x$ & $M x$ & $\neg C x$ \\
\hline 6 & $\neg M x$ & $\neg C x$ & $M x$ & $C x$ & 14 & $\neg M x$ & $C x$ & $\neg M x$ & $\neg C x$ \\
\hline 7 & $\neg M x$ & $C x$ & $\neg M x$ & $C x$ & 15 & $M x$ & $\neg C x$ & $\neg M x$ & $\neg C x$ \\
\hline 8 & $\neg M x$ & $C x$ & $M x$ & $\neg C x$ & 16 & $\neg M x$ & $\neg C x$ & $\neg M x$ & $\neg C x$ \\
\hline
\end{tabular}

Tomemos a linha 7. Ela significa um estadow $\in W$ onde a é não é mulher, nem casado e b é mulher e casada: $\neg M(a) \wedge \neg C(a) \wedge M(b) \wedge C(b)$. Já uma afirmação $i$ que expresse algo como " $a$ é mulher ou casado e $b$ é mulher e casada": $((M(a) \vee C(a)) \wedge(M(b) \wedge C(b)))$ poderia ser satisfeito por 3 dos 16 estados possíveis, nomeadamente, os mundos $\mathrm{w}_{1}, \mathrm{w}_{2}$ e $\mathrm{w}_{3}$. Portanto a confirmação de i elimina 13 dos 16 estados de descrição.

Mas, o conceito (função) Cont não é capaz de definir sem ambiguidade a soma de informação entre mensagens. Daí, Carnap e Bar-Hillel introduzirem o conceito pré-sistemático de quantidade de informação in (com i minúsculo). Este conceito permite realizar operações algébricas com a quantidade de informação entre mensagens. Mas, há casos em que a soma pode ser menor que a conjunção entre os conteúdos. (cf. BAR-HILLEL; CARNAP, 1952. pp.11-13.)

A partir das definições Cont $\mathrm{e}$ in, eles derivam a medida quantitativa cont (medida de conteúdo), uma medida que se baseia na medida de probabilidade $\mathrm{m}()$, e esta probabilidade é definida a priori (podendo ser estabelecida segundo outros critérios, e.g. estatísticos, indutivos, por distribuições assimétricas, etc.). Note-se que $\mathrm{W}$ é definido para ter estados equiponderáveis e que, para toda sentença i, há um conjunto de estados de W que torna i verdadeira (no exemplo anterior: $\mathrm{w}_{1}, \mathrm{w}_{2}$ e $\left.\mathrm{w}_{3}\right)$ e outro conjunto de estados de $\mathrm{W}$ que torna ifalsa $\operatorname{Cont}(i)=$ $\{w 4, \ldots, w 16\})$ ainda que, tal conjunto possa ser vazio, ou o próprio W.Assim, toda sentença de L pode ser associada a uma probabilidade $\mathrm{m}()$, sendo $\mathrm{m}()$ calculada a partir da probabilidade de um conteúdo i, em que m()é medido pela soma de todos os estados de descrição de i, tais que pertençam à $\mathrm{R}(\mathrm{i})$, onde $\mathrm{R}$ é o Range de $\mathrm{i}$, i.e. todos os estados que tornam $\mathrm{i}$ verdadeiro. $\mathrm{O}$ limite do valor absoluto de $\mathrm{m}()$ é a soma das probabilidades de todos estados possíveis de $\mathrm{W}$ que é 1 . A partir daí, temos a função:

(2) $\operatorname{cont}(i)=1-m(i)^{2}$

Esta função nos permite medir a quantidade relativa de possibilidades excluídas pela proposição $i$. Note que $1 \geq \operatorname{cont}(i) \geq 0$ se $i$ é contingente, $\operatorname{cont}(i)=1$ se $i$ é uma contradição e $\operatorname{cont}(i)=0$ se $i$ é uma tautologia. Assim, seja $m(i)=1 / 3$ para a ocorrência da sentençai, então seu conteúdo nos fornece um valor de $2 / 3$, o que significa que $2 / 3$ dos estados de $\mathrm{W}$ são

${ }^{2}$ Alternativamente, $\mathrm{m}(\mathrm{i})=1$-cont(i) e cont $(\neg \mathrm{i})=\mathrm{m}(\mathrm{i}) .($ Cf. BAR-HILLEL; CARNAP, 1956. p.15) 
excluídos em razão da ocorrência de $i$. Isto é, cont é a medida de probabilidade de todos os estados que não satisfazem certo conteúdo: $\operatorname{cont}(i)=m(\neg i)=1-m(i)$.

Citaremos algumas das relações fundamentais de probabilidade entre subfórmulas atômicas $i$ e $j$ de uma fórmula molecular:

(a) $m(i \wedge j)=m(i) \times m(j)$ sss $i$ e $j$ não têm predicados em comum.

(b) $m(i \wedge j) \leq m(i) \leq m(i \vee j)$

(c) $m(i \vee j)=m(i)+m(j)-m(i \wedge j)$

$(\mathrm{d}) m(i \vee j)=m(i)+m(j)$ sssi $\wedge j$ é umacontradiçãoemL

(e) $m(i \wedge j)=m(i)+m(j)-m(i \vee j)$

(f) $m(i \wedge j)=m(i)+m(j)-1$ sssi $\vee j$ é umatautologiaemL

De acordo com o exemplo $(\operatorname{Ex} 1) i=((M(a) \vee C(a)) \wedge(M(b) \wedge C(b)))$, i satisfaz 3 de 16 possibilidades, seja $i=\left(\left(j^{\prime} \vee j^{\prime \prime}\right) \wedge\left(k^{\prime} \wedge k^{\prime \prime}\right)\right), m\left(j^{\prime} \vee j^{\prime \prime}\right)=m\left(j^{\prime}\right)+m\left(j^{\prime \prime}\right)-m\left(j^{\prime} \wedge\right.$ $\left.j^{\prime \prime}\right)$ Como $j^{\prime} \vee j^{\prime \prime}$, não possuem predicados em comum, $m\left(j^{\prime} \vee j^{\prime \prime}\right)=m\left(j^{\prime}\right)+m\left(j^{\prime \prime}\right)-$ $\left(m\left(j^{\prime}\right) \times m\left(j^{\prime \prime}\right)\right)=\frac{1}{2}+\frac{1}{2}-\frac{1}{4}=\frac{3}{4}$ e $m\left(k^{\prime} \wedge k^{\prime \prime}\right)=m\left(k^{\prime}\right) \times m\left(k^{\prime \prime}\right)=\frac{1}{4} ; \log o, m\left(\left(j^{\prime} \vee\right.\right.$ $\left.\left.j^{\prime \prime}\right) \wedge\left(k^{\prime} \wedge k^{\prime \prime}\right)\right)=\frac{3}{4}+\frac{1}{4}-\left(\frac{3}{4}\right)=\frac{1}{4} \operatorname{assim}, m(i)=\frac{1}{4}$ e portanto, dizemos que $\operatorname{cont}(i)=1-$ $\frac{1}{4}=\frac{3}{4}$

Uma intuição importante sobre a informaçãorepresentada por sentenças é a de que, se i e j são sentenças indutivamente independentes ${ }^{3}$, a quantidade de informação de $i \wedge j$ deveria ser igual à quantidade de informação de i mais a quantidade de informação de j, isto é, uma função da quantidade de informação semântica deveria ser aditiva sob a conjunção quando as sentenças são indutivamente independentes. Um problema da função cont é que ela não respeita essa intuição, pois, $\operatorname{cont}(i \wedge j) \leq \operatorname{cont}(i)+\operatorname{cont}(j)$.

(Ex2): Suponha a proposição i e $\mathrm{j}$ como " $a$ e $b$ são mulheres": $M(a) \wedge M(b)$.Então, $\operatorname{cont}(i \wedge j) \leq \operatorname{cont}(i)+\operatorname{cont}(j) \Leftrightarrow \operatorname{cont}(i)+\operatorname{cont}(j)-\operatorname{cont}(i \vee j) \leq$ $\operatorname{cont}(i)+\operatorname{cont}(j) \Rightarrow \frac{1}{2}+\frac{1}{2}-\frac{1}{4} \leq \frac{1}{2}+\frac{1}{2} \Rightarrow \frac{3}{4} \leq 1$.

Outra questão é que segundo a definição de cont, a expressão cont( $\mathrm{i}->\mathrm{j})$ ou $\operatorname{cont}(\mathrm{j} / \mathrm{i})=1 / 2$ cont(i) ao invés de calcular a informatividade de j e de i, remove j do cálculo. Para resolver ambos os problemas, Carnap propõe uma função que preserve o aspecto informativo de cada

${ }^{3}$ Definimos independência indutiva entre duas sentenças quando a probabilidade de uma sentença, dada a ocorrência da outra sentença, permanece a mesma. Em contraste, a independência de conteúdo ocorre quando, dadas as sentenças i e j, o conteúdo semântico de i é disjunto ao conteúdo semântico de j. Isto é, i e j são independentes com relação ao conteúdo se $\operatorname{Cont}(\mathrm{i}) \cap \operatorname{Cont}(\mathrm{j})=\varnothing$. 
sentença, quando elas são expressas em conjunto ou quando uma se segue da outra, introduzindo a função inf (medida de informação). Tal que:

(4) $\inf (i)=\log _{2} \frac{1}{1-\operatorname{cont}(i)}=\log _{2} \frac{1}{m(i)}=-\log _{2} m(i)$

Isto permite calcular $\inf (q \rightarrow p)=\inf (p \wedge q)-\inf (q)=-\log m(q \rightarrow p)$.

Sendo o limite de inf para sentenças contingentes variando de maior que 0 , até menor que infinito.Vejamos um exemplo envolvendo (3) e uma das sentenças anteriores:

(Ex3): como já dispomos do valor de $i=((M(a) \vee C(a)) \wedge(M(b) \wedge C(b))), m(i)=$ $\frac{1}{4} \operatorname{cont}(i)=\frac{3}{4}$, então temos que $\inf (i)=\log _{2} \frac{1}{1-\frac{3}{4}}=\log _{2} \frac{1}{\frac{1}{4}}=-\log _{2} \frac{1}{4}=2$

Isto significa dizer que a mensagem de $i$ possui dois bits de informação. Cada bit de informação corresponde a uma escolha entre duas opções disponíveis. Em nosso caso, há prédeterminado 16 resultados equiprováveis (os 16 mundos possíveis envolvendo a combinação dos indivíduos com os predicados), onde, para uma resposta precisa de qual das 16 opções é o caso, seria necessário um total de 4 bits, i.e. uma sentença $j$ qualquer, que expressasse precisamente uma das 16 opções, deveria resulta em $\inf (j)=4$.A sentença $i$ do exemplo, expressa 2 bits deles. Isto significa que a sentença aumenta a certeza (i.e. reduz as possibilidades) de 16 opções, para 4 opções.

Já a função $m(i)$ retorna a probabilidade de i ser o caso, uma vez que, inf $(i)$ retorna a quantidade de redução da incerteza acerca de qual $w$, tal que $w \in W$, é o caso, dada uma afirmação i. Ou seja, qual dos estados descritos em L é o caso. Note que, para tautologias, $\inf (T)=0$ e $\operatorname{cont}(T)=0$ já que $m(i)=1 \mathrm{em}(T)=1 \mathrm{e}$ para contradições $\inf (\perp)=\infty$ e $\operatorname{cont}(\perp)=1$, já que $m(i) \cong 0$ e $m(\perp)=0$.

A segunda vertente da teoria da informação semântica, a Teoria da Informação Semântica Forte (TSSI) propõe novas grandezas e novas medidas para a mensuração da informação semântica, ela postula que a informação só pode ser definida enquanto tal, na medida em que encapsula o valor de verdade "verdadeiro". A TSSI possui os seguintes desiderata: 1. Evitar inconsistências como o Paradoxo Bar-Hillel/Carnap (BCP). Fornecendo um modo de reinterpretar ${ }^{4}$ as funções construídas por Carnap e Bar-Hillel, atribuindo a elas um novo papel informativo, propondo novas grandezas e medidas resultantes. 2. Estabelecer o valor verdadeiro como uma característica necessária para a análise quantitativa da informação. 3 . Estender a análise quantitativa à toda família de conceitos relacionados à informação como

\footnotetext{
${ }^{4}$ Como a questão sobre sentenças falsas não é de utilidade para o nosso estudo, não entraremos em pormenores do assunto. Para uma interpretação das equações de Carnap e Bar-Hillel pela TSSI de modo a superar o BCP, cf. FLORIDI, 2011, pp.127-129.
} 
informatividade, desinformação, informação falsa, vacuidade e imprecisão de informação semântica. (cf. FLORIDI, 2011, p.117).

É evidente que o modo como contradições lógicas são integradas (ou expulsas) de dada teoria da informação semântica é um fator determinante na definição das teorias fraca e forte da informação semântica. As contradições lógicas não são informativas (TSSI) ou informativas de mais para serem verdade (TWSI). Mas, e o outro extremo das sentenças lógicas? Como essas teorias interpretam as tautologias? Para TWSI e TSSI tautologias não têm utilidade informacional. Devemos perguntarem que sentido tautologias (sentenças L-verdadeiras)não possuem valor informativo. Para Carnap e Bar-Hillel:

[...] ocorrerá, sob todas as explicações previstas por nós, que a quantidade de informação transportada pela sentença '17 X $19=323$ 'é zero e que a quantidade de informação de 'As três medianas dos lados de um triângulo plano se cruzam em um só ponto', relativa a um conjunto de frases que servem como um conjunto completo de axiomas para a geometria euclidiana, é igualmente zero. Isso, no entanto, não deve ser de forma alguma entendidocomo a implicação de que não há uma 'quantidade de informação" em bom sentido, no qual a quantidade de informação dessas frases não será, de fato, zero, e para algumas pessoas, pode até ser bastante alta. Para evitar ambiguidades, vamos usar o adjetivo "semântica" para diferenciar ambos os sentidos pré-sistemáticos de "informação" em que estamos interessados no momento e sua explicata sistemática de outros sentidos (tais como "quantidade de informações psicológica para a pessoa P" ) e os seus explicata. Este adjetivo vai, no entanto, ser deixado, em casos em que ambiguidades são pouco prováveis de ocorrer. (CARNAP; BAR-HILLEL, 1952, p.3).

Para eles, portanto, sentenças do domínio da lógica e da matemática não possuem informação semântica, a despeito de que possa acarretar certa quantidade de informação psicológica para um sujeito S. Ora, penso que ocorra aqui uma confusão entre psicológico e epistêmico, que acaba por ocultar o fato de que sentenças oriundas de deduções podem produzir informação nova. O conhecimento de ordem psicológica, diz respeito ao conjunto de informações que um indivíduo particular possui baseado em suas vivências. O conhecimento de ordem epistêmica diz respeito à informação acerca das estruturas do conhecimento, publicamente compartilhável entre os falantes. Uma distinção já antevista por Frege (2009. p.33-39) e explicitamente demarcada por Wittgenstein (viz. distinção entre Darstellung e

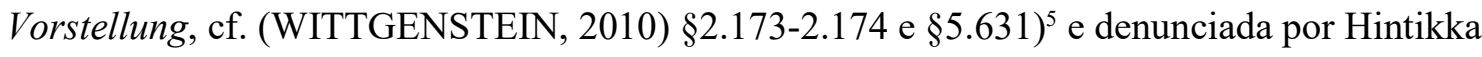
(1970, p.288-291) através da diferença entre o aspecto psicológico e o aspecto lógico do conhecimento. O aspecto psicológico, ou a representação subjetiva das coisas (Vorstellung) é o

\footnotetext{
${ }^{5}$ D'Agostino e Floridi apontam uma resposta de Wittgenstein no Tractatus ao problema da informatividade de sentenças analíticas (cf. WITTGENSTEIN, 2010, §5.13; §6.122; §6.127): "as tautologias são autoevidentes, basta uma inspeção de sua estrutura e a tautologia é revelada". Mas, a resposta é insuficiente por dois motivos: primeiro, como bem aponta Hintikka, nem sempre uma simples inspeção revela a tautologia, segundo, este modo de tratamento só se aplica a lógicas decidíveis, como o caso da LP. (Cf. D’AGOSTINO; FLORIDI, 2008, pp.8-9.)
} 
que podemos definir como um conhecimento relativo a um sujeito $S$ a partir de suas impressões e vivências. $\mathrm{O}$ aspecto lógico, que é intersubjetivamente acessível, julgável e asserível é constitutivo da representação objetiva (Darstellung).

Mesmo no caso de considerar a lógica apenas como instrumental prioritário de abordagem da realidade ${ }^{6}$ ou acatando a tese epistemológica da inacessibilidade à forma da realidade mesma, o emprego da lógica e da matemática fornece um valor informativo epistêmico, acerca de certa forma atualmente válida de representação das coisas (Darstellung). Em última instância, a negação do valor informativo epistêmico das sentenças analíticas, reduziria à zero o papel informativo do próprio trabalho de Carnap e Bar-Hillel. Pois, eles descrevem certo modo de apresentar a informação, certa norma para se computar quantidade de informação. Ora, o que fazem em seu texto, de fato, não é descrever coisas, i.e. nos informar no sentido de dizer que situações são ou não o caso. Mas, nos fornecer um modo eficiente de representar a própria informação possível acerca das situações. E esta argumentação deve ser composta de sentenças analíticas.

\section{CONCLUSÃO}

Poderíamos objetar que o valor informativo é útil para nos dar clareza psicológica sobre o assunto do texto. O que podemos chamar de informação psicológica (PI). De fato, esta classe de informação é importante (em contextos singulares) e não pode ser ignorada ao falarmos do processo de aquisição e exercício de nosso conhecimento. Mas, note que informação psicológica, nestes termos, diz respeito à clareza de nossas representações pessoais ao expressarmos elas a outrem. E, portanto, tratando-se de informações acerca de nossas representações. A adoção irrefletida desta tese nos levaria à separação entre nossas representações, a informatividade delas e as coisas mesmas. A aceitação dela aponta para um abismo epistemológico entre as coisas e o que sabemos delas ${ }^{8}$. Se com o auxílio da lógica e da matemática construímos e adquirimos conhecimento sobre o que sabemos e sobre a forma atualmente aceita da própria realidade, não há razão suficiente para negar o valor informativo

\footnotetext{
${ }^{6}$ i.e.a lógica como um instrumental de aporte epistêmico. O que seria um enfraquecimento da tese de que o estudo da lógica, de algum modo, desvela (ou é a própria) a estrutura última da realidade, tese característica de Frege e Wittgenstein (à época do Tractatus).

${ }^{7}$ Como veremos a seguir, certas regrasmateriais de inferência.

8 Claro que uma alternativa para evitar o abismo seria dizer que toda representação é publica e portanto intersubjetivamente acessível. Mas, isto seria afirmar que a mente é um produto exclusivamente linguístico (remontando ao behaviorismo brando de Gilbert Ryle). Em princípio, recusaremos esta proposta, uma vez que, associa necessariamente comportamento e mente. Para maiores discussões (e.g. cf. MASLIN, 2009.; CHURCHLAND, 2004.; RYLE, 1949. CUMMINS, 1991.)
} 
das sentenças analíticas. Portanto, a atividade de deduzir, isto é, de produzir novas sentenças via dedução, produz informação objetiva nova, quer da perspectiva epistemológica, quer da perspectiva ontológica.

Uma boa prova para este argumento é o problema apontado por (JUHL; LOOMIS, 2010. p.59-60). Nos termos da teoria da informação de Bar-Hillel e Carnap, a principal definição de tautologia é uma definição semântica. Isto significa que uma sentença p qualquer é uma tautologia, se ela é satisfeita em todos os estados de descrição possíveis de L, portanto, não é informativa. Para eles, L-verdade, logicamente verdadeiro, analítico e tautologia (e.g. $P a \vee$ $\neg P a$ ) estão na mesma categoria (cf. CARNAP; BAR-HILLEL, 1952, p.4).

Entretanto, tomemos o exemplo (Ex1). Se uma sentença deve ser satisfeita por todos os estados de descrição de uma Linguagem, a completude desta propriedade deveria conter todas as combinações de tal forma que os predicados da linguagem devem ser tomados independentemente.

Há duas evidências em favor do aspecto informativo das sentenças analíticas contidas na explicação acima. A primeira é a introdução de predicados como $C x$ e $S x$ para x é Casado e x é Solteiro. Note que a introdução direta desse vocabulário em L levaria a uma contradição em termos extencionais ${ }^{9}$, mas não uma contradição sintática. Para evitar isto, os autores estabeleceram (implicitamente) uma regra de equivalência, por exemplo: $C x \equiv \neg S x$. (cf. CARNAP; BAR-HILLEL, 1952, p.5). Entretanto, a estipulação dessa regra pressupõe mais do que a simples análise dos predicados poderia fornecer. Mostrando que colocar os conceitos logicamente verdadeiro, analítico e tautologia equivalente ao conceito de L-verdadeiro é extremamente forçoso. Pois, sintaticamente falando, não há semelhança entre $C x \equiv \neg S x$ e $C a \vee$ $\neg$ Ca. O segundo problema diz respeito à aplicação artificial da definição de L-verdadeiro por satisfação de estados de descrição. Por exemplo, tomemos os predicados $R x$ e $A x$ para, x é Verde e x é Amarelo, respectivamente. Note que haverá em alguns estados de descrição da linguagem $\mathrm{L}$, algo como $R a \wedge A a$. Tomemos $a$ como determinado ponto dotado de coordenadas espaço-temporais precisas, intrínsecas na sua descrição. O que a expressão $R a \wedge A a$ significaria, portanto, é que determinado ponto, em determinadas coordenadas, é verde e amarelo ao mesmo tempo. O que consiste em uma contradição. Exigindo a introdução a priori

\footnotetext{
${ }^{9}$ A crítica de Quine sobre a condição suficiente para a analiticidade ser determinada a partir da coextencionalidade incide diretamente sobre este ponto (cf. QUINE, 2010. pp.50-52). Tomemos por exemplo: todo animal com rim e todo animal com coração. Estas expressões são coextencionais, mas estão longe de ser interdefiníveis, analíticas ou qualquer coisa que nos leve a chamar de verdadeiras em $L$ em função se deu significado. Exceto se estipularmos a priori, uma relação de equivalência entre elas, mas como veremos a seguir, isto a coloca em outra classe de sentenças.
} 
de algumas regras que servirão de limitação (constraint) para certas combinações de predicados e constantes de L. O que demonstra que as sentenças analíticas em Lsão de fato informativas, em especial sobre os limitadores (ou regras de apresentação, ou estipulações a priori) da nossa linguagem L e do mundo que se pretende descrever, ao contrário do que pode parecer à primeira vista.

É interessante notar que não é apenas possível e expediente introduzir equivalências entre termos. Mas, outras classes de expressões lógicas devem figurar a lista de relações a priori em uma linguagem que pretenda descrever algum subconjunto de entidades ou fenômenos do mundo. Pagarei emprestado a descrição feita por Brandom de um conjunto de expressões que figuram uma inferência material:

Considere os argumentos que estão codificados nas seguintes condicionais:

1 Se eu risco este fósforo seco e bem feito, então ele irá acender. $(p \rightarrow q)$

2 Se $\mathrm{p}$ e o fósforo esta em um campo eletromagnético muito forte, então ele não irá acender. $(p \& r \rightarrow \sim q)$

3 Se p e r e o fósforo está emu ma gaiola de Faraday, então ele irá acender ( $p \& r \& s$ $\rightarrow q)$

4 Se p e r e s e a sala está vazia de oxigênio, então ele não irá acender. If $p$ and $r$ and $s$ and the room is evacuated of oxygen, then it will not light. ( $p \& r \& s \& t \rightarrow \sim q$ )

O raciocínio em que nos envolvemos sempre permite a construção de hierarquias inferenciais com conclusões oscilantes como esta (BRANDOM, 2000. p.88).

É a isto que também Sellars chama de princípio material de inferência (SELLARS, 1953). Neste sentido, é possível interpretar tais condições descritas acima $C x \equiv \neg S x$ como expressões baseadas em inferências materiais que devem ser adotadas ao se abordar determinado domínio. Assim, dada uma lista a priori de inferências materiais poderemos calcular o grau de informatividade de uma sentença que figure nestas regras, não como sentenças contingentes. Mas, como sentenças analíticas dotadas de informação. Embora o cálculo seja essencialmente o mesmo, o significado do valor informacional é outro. Uma vez que ele representa o grau de restrição com o qual o mundo deve ser representado. Inclusive em situações de predicados materialmente excludentes, como é o caso da citação de Brandom.

O impacto desta classes de sentenças é mínimo. Basta adicionar ao cálculo de uma sentença $\alpha$ qualquer sua conjunção com as sentenças que representam estas inferências materiais, digamos uma sentença $\varphi$. Assim, obtendo:

(5) $\operatorname{cont}(\alpha \wedge \varphi)=1-m(\alpha \wedge \varphi)$

Note que agora o cálculo de conteúdo de uma expressão passaria a ser feito com base na pressuposição de que todas as inferências materiais deste domínio já estejam satisfeitas.

(6) $\operatorname{Cont}(\alpha)=\{\mathrm{w} \in \mathrm{W} \mid w \vDash(\neg \alpha) \wedge \varphi\}$, 
Isto significa dizer que o cálculo de conteúdo deve ser feito sempre pressupondo que tais e tais expressões $\varphi$ sejam verdadeiras. Da perspectiva da informatividade de $\alpha$, temos a impressão de que $\varphi$ é uma tautologia. Mas isto se prova errado se executarmos os cálculos (1) - (4) em $\varphi$ isoladamente. O que nos permite modelar e diferenciar a informatividade das inferências não-lógicas (materiais) e a informatividade de expressões factuais. 


\section{REFERENCIAS}

ADRIAANS, P.; VAN BENTHEM, J. Introduction: Information is what Information does. In: Adriaans, P.; van Benthem, J. (Eds.). Handbook of Philosophy of Science. Netherlands: Elsevier B. V., 2008. v. 8: Philosophy of Information.

AUSTIN, J. L. How to do Things with Words. Oxford: Clarendon Press, 1962.

BAR-HILLEL, Y. Language and Information: selected essays on their theory and application. Jerusalem: Jerusalem Academic Press, 1964.

Press, 1952.

; CARNAP, R. An outline of a theory of semantic information. Massachusetts: MIT

BRANDOM, R. B. Articulating reasons: an introduction to inferencialism. Cambridge: Harvard University Press, 2000.

Making it explicit: reasoning, representing and discursive commitment. Cambridge: Harvard University Press, 1998.

BREMER, M. E. Do logical truths carry information? Minds and Machines, v. 13, p. 567 575, 2003.

BURGIN, Mark. Theory of information: fundamentality, diversity and unification.

Singapore: World Scientific Publishing Company, 2010.

CARNAP, R. Logical foundations of probability. Chicago: University of Chicago Press, 1950.

The two concepts of probability: the problem of probability. Philosophy and Phenomenological Research, v. 5, n. 4, p. 513-532, 1945.

The logical Syntax of language. London: Routledge, 1949.

Two essays on entropy. Los Angeles: University of California Press, 1977.

CHAITIN, G. J. Algorithmic information theory. 3. ed. Cambridge: Cambridge University Press, 1997.

From philosophy to program size, key ideas and methods: lecture notes on algorithmic information theory. Tallinn: Institute of Cibernetics, 2003.

CHERRY, C. On human communication. Massachusetts: M. I. T. Press, 1957.

D'AGOSTINO, M.; FLORIDI, L. The enduring scandal of deduction: is propositional logic really uninformative? Netherlands: Kluwer Academic Publishers, 2008.

DOUSA, T. M.; IBEKWE-SANJUAN, F. Studies in history and philosophy of science: theories of information, communication and knowledge: a multidisciplinary approach. New York: Springer, 2014. 
DRETSKE, F. Knowledge and the flow of information. Stanford: CSLI Publications, 1999.

ENDERTON, H. B. A mathematical introduction to logic. Los Angeles: Harcourt Academic Press, 2001.

FLORIDI, L. The philosophy of information. Oxford: Oxford University Press, 2011. 2014. ; ILLARI, P. (Eds.) The philosophy of information quality. New York: Springer,

FREGE, G. Os Prmeiros escritos lógicos de Gottlob Frege. Trad. Paulo Alcoforado; Alessandro Duarte; Guilherme Wyllie. São Paulo: Instituto Brasileiro de Filosofia e Ciência Raimundo Lúlio "Ramon Llull", 2012.

. Lógica e filosofia da linguagem. Trad. Paulo Alcoforado. São Paulo: Edusp, 2009.

. Os fundamentos da aritmética: uma investigação lógico-matemática sobre o conceito de número. Trad. Org. Luís H. L. dos Santos. São Paulo: Abril Cultural, 1983.

HEMPEL, C. G. On Nature of Mathematical Truth. American Mathematical Monthly. Washington: Mathematical Association of America, 1945. v. 52, p.543-556,

HINTIKKA, J. Information, Deduction, and the A Priori. Noûs, v. 4, n. 2, p. 135-152, 1970. 1970. ; SUPPES, P. Information and inference. Dordrecht: D. Reidel Publishing Company, 34

JUHL, C.; LOOMIS, E. Analiticity. New York: Routledge, 2010.

MASLIN, K. T. Introdução à Filosofia da Mente. Porto Alegre: Artmed, 2009

PEREIRA, L. C.; HAEUSLER, E. H.; PAIVA, V. de. (Eds.) Advances in natural deduction: A Celebration of Drag Prawitz's Work. Dordrecht: Springer, 2014.

QUINE, W. V.O. De um ponto de vista lógico. São Paulo: Unesp, 2010.

RYLE, G. The Concept of Mind. New York: Barnes and Noble, 1949

SELLARS, W. Inference and meaning. Mind, v. 62, n. 247. p. 313-338, 1953.

SEQUOIAH-GRAYSON, S. Information flow and impossible situations. Logique et Analyse, v.196, p. 371-398, 2006.

SHANNON, C. E. Collected papers. New York: IEEE Press, 1993.

WITTGENSTEIN, L. Philosophical Investigations. 4. ed. Oxford: Blackwell, 2009. . Tratado lógico filosófico. Edição bilíngue. São Paulo: Edusp, 2010. 